\title{
Transitive groups of axial homologies of hyperbola structures and Minkowski planes
}

\author{
Hans-Joachim Kroll Andrzej Matraś*
}

\begin{abstract}
In this paper a typification of the automorphism groups of hyperbola structures based on the notion of axial homologies (i.e. automorphisms fixing two generators of the same kind) is given. For the class of hyperbola structures over half-ordered fields (cf. [6, 12]) the types of the full automorphism groups are determined.
\end{abstract}

Keywords : axial homology, hyperbola structure, Minkowski plane

MSC 2000: 51B20

\section{Introduction}

Analogous to the well known Lenz-Barlotti typification of projective planes there are typifications for Minkowski planes based on the notion of $G$ - and $p$-translations and $(p, q)$-homologies (cf. [9, 8]). The typification with respect to transitive groups of $G$-translations was done actually for the more extensive class of hyperbola structures (cf. [9]). Beside the $G$-translations axial homologies can be defined for hyperbola structures. An automorphism $\alpha$ of a hyperbola structure $\mathcal{H}$ is called a $(G, H)$-homology if $\alpha$ fixes pointwise the generators $G, H$ (where $G \cap H=\emptyset$ ). Let $\mathrm{A}(G, H)$ denote the group of all $(G, H)$-homologies. For a given subgroup $\Gamma$ of the automorphism group of $\mathcal{H}$ we are interested in the configuration $\mathfrak{A}(\Gamma)$ of all 2-sets $\{G, H\}$ of generators such that the group $\mathrm{A}(G, H) \cap \Gamma$ acts transitively on $X \backslash(G \cup H)$ for a generator $X$. By determining all possible configurations $\mathfrak{A}(\Gamma)$ we obtain a typification for the automorphism groups of hyperbola structures (cf. Theorem 3.3). In the case of a hyperbola

\footnotetext{
*This research was supported in part by DAAD.
} 


\begin{abstract}
1
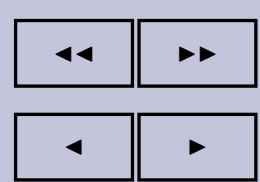

page $2 / 18$

go back

full screen
\end{abstract}

close

quit

\title{
2. Hyperbola structures: definitions and notations
}

Hyperbola structures can be defined in different ways (cf. [5, 1, 7]). For our purpose the representation by permutation sets is most suitable. Let $(M, \Sigma)$ be a permutation set ${ }^{1}$. Putting $P:=M \times M, \mathfrak{K}:=\{\{(x, \gamma(x)) \mid x \in M\} \mid \gamma \in \Sigma\}$ and $\mathfrak{G}_{1}:=\{\{a\} \times M \mid a \in M\}, \mathfrak{G}_{2}:=\{M \times\{a\} \mid a \in M\}$ we obtain an incidence structure $\mathcal{H}(M, \Sigma)=\mathcal{H}(\Sigma):=\left(P, \mathfrak{K}, \mathfrak{G}_{1} \cup \mathfrak{G}_{2}\right)$; the elements of $P$, $\mathfrak{K}$ and $\mathfrak{G}:=\mathfrak{G}_{1} \cup \mathfrak{G}_{2}$ are called points, chains and generators respectively, and $n:=|M|-1$ is called the order of $\mathcal{H}(M, \Sigma)$. For every point $x \in P$ and $i \in\{1,2\}$ there is exactly one generator $X \in \mathfrak{G}_{i}$ with $x \in X$ (notation: $[x]_{i}:=X$ ). Then $\mathcal{H}(M, \Sigma)$ is called a hyperbola structure, if $\Sigma$ is acting sharply 3-transitively on $M$. The chains of a hyperbola structure are called circles.

Let $\mathcal{H}=\left(P, \mathfrak{K}, \mathfrak{G}_{1} \cup \mathfrak{G}_{2}\right)$ be a hyperbola structure. For every point $w \in P$ we define: $[w]:=[w]_{1} \cup[w]_{2}, P^{w}:=P \backslash[w], \mathfrak{K}(w):=\{X \in \mathfrak{K} \mid w \in X\}$, $\mathfrak{K}^{w}:=\{X \backslash\{w\} \mid X \in \mathfrak{K}(w)\}, \mathfrak{G}_{i}^{w}:=\left\{X \cap P^{w} \mid X \in \mathfrak{G}_{i}, w \notin X\right\}$ for $i \in\{1,2\}$, and $\mathcal{H}^{w}:=\left(P^{w}, \mathfrak{K}^{w}, \mathfrak{G}_{1}^{w} \cup \mathfrak{G}_{2}^{w}\right), \mathcal{A}(w):=\left(P^{w}, \mathfrak{K}^{w} \cup \mathfrak{G}_{1}^{w} \cup \mathfrak{G}_{2}^{w}\right)$. Then $\mathcal{H}^{w}$ is called the derivation of $\mathcal{H}$ in the point $w$. Moreover, $\mathcal{H}$ is called a Minkowski plane, if $\mathcal{A}(w)$ is an affine plane for all $w \in P$; in this case $\mathcal{A}(w)$ is called the affine derivation of $\mathcal{H}$ in $w$.

Let $\mathcal{H}(M, \Sigma)$ and $\mathcal{H}\left(M^{\prime}, \Sigma^{\prime}\right)$ be two hyperbola structures and $\varphi: P \rightarrow P^{\prime}$ a bijection. The bijection $\varphi$ is called an isomorphism from $\mathcal{H}(M, \Sigma)$ onto $\mathcal{H}\left(M^{\prime}, \Sigma^{\prime}\right)$ if $\varphi(\mathfrak{K})=\mathfrak{K}^{\prime}$.

Let $\varphi$ be an isomorphism. Then $\varphi\left(\mathfrak{G}_{1}\right)=\mathfrak{G}_{1}^{\prime}, \varphi\left(\mathfrak{G}_{2}\right)=\mathfrak{G}_{2}^{\prime}$ or $\varphi\left(\mathfrak{G}_{1}\right)=\mathfrak{G}_{2}^{\prime}$, $\varphi\left(\mathfrak{G}_{2}\right)=\mathfrak{G}_{1}^{\prime}$.

\footnotetext{
$1_{\text {i.e. }} \Sigma$ is a set of permutations of the set $M$
} 
For every isomorphism $\varphi$ there exist two bijections $\alpha, \beta: M \rightarrow M^{\prime}$, such that $\varphi(x, y)=(\alpha(x), \beta(y))$ for all $(x, y) \in P$ or $\varphi(x, y)=(\alpha(y), \beta(x))$ for all $(x, y) \in P$.

Lemma 2.1 (Wefelscheid [14]). Let $\mathcal{H}(M, \Sigma)$ and $\mathcal{H}\left(M^{\prime}, \Sigma^{\prime}\right)$ be two hyperbola structures and $\alpha, \beta: M \rightarrow M^{\prime}$ two bijections.

(1) $\varphi:(x, y) \mapsto(\alpha(x), \beta(y))$ is an isomorphism iff $\Sigma^{\prime}=\beta \Sigma \alpha^{-1}$.

(2) $\varphi:(x, y) \mapsto(\alpha(y), \beta(x))$ is an isomorphism iff $\Sigma^{\prime}=\beta \Sigma^{-1} \alpha^{-1}$.

full screen

close

An automorphism $\varphi$ of a hyperbola structure $\mathcal{H}$ is called proper, if $\varphi\left(\mathfrak{G}_{1}\right)=\mathfrak{G}_{1}$.

The following notation is convenient to describe the proper automorphisms of $\mathcal{H}(M, \Sigma)$. For $\alpha \in \operatorname{Sym} M$ let denote $\alpha: M \times M \rightarrow M \times M, \quad(x, y) \mapsto(\alpha(x), y)$ and $\grave{\alpha}: M \times M \rightarrow M \times M, \quad(x, y) \mapsto(x, \alpha(y))$. For every proper automorphism $\varphi$ there exist $\alpha, \beta \in \operatorname{Sym} M$ with $\varphi=\dot{\alpha} \circ \grave{\beta}$.

The classical model of a Minkowski plane is $\mathcal{H}(\operatorname{PGL}(2, \mathbb{F}))$, where $\mathbb{F}$ is a commutative field. Every Minkowski plane isomorphic to a plane $\mathcal{H}(\operatorname{PGL}(2, \mathbb{F}))$ is called a Miquelian Minkowski plane.

Modifying the classical model J. Jakobowski [6] constructed a class of nonMiquelian Minkowski planes. Let $(\mathbb{F}, P)$ be a half-ordered field, i.e. $P$ is a subgroup of index 2 of the multiplicative group $\left(\mathbb{F}^{*}, \cdot\right)$ of the field $(\mathbb{F},+, \cdot)$. Let $\chi: \mathbb{F}^{*} \rightarrow\{-1,1\}$ denote the character belonging to $P, \overline{\mathbb{F}}=\mathbb{F} \cup\{\infty\}$ the projective line over $\mathbb{F}$ and

$$
\overline{\mathbb{F}}^{(3)}:=\left\{\left(x_{1}, x_{2}, x_{3}\right) \in \overline{\mathbb{F}}^{3}||\left\{x_{1}, x_{2}, x_{3}\right\} \mid=3\right\} .
$$

For $\left(x_{1}, x_{2}, x_{3}\right) \in \overline{\mathbb{F}}^{(3)}$ we define $\omega\left(x_{1}, x_{2}, x_{3}\right)=\chi\left(x_{1}-x_{2}\right) \chi\left(x_{2}-x_{3}\right) \chi\left(x_{3}-x_{1}\right)$ where $\chi(x-y):=\left\{\begin{aligned} 1 & \text { for } x=\infty \\ -1 & \text { for } y=\infty\end{aligned}\right.$.

A permutation $f \in \operatorname{Sym} \overline{\mathbb{F}}$ is called order-preserving (order-reversing) if for all $\left(x_{1}, x_{2}, x_{3}\right) \in \overline{\mathbb{F}}^{(3)}$,

$$
\omega\left(f\left(x_{1}\right), f\left(x_{2}\right), f\left(x_{3}\right)\right)=\omega\left(x_{1}, x_{2}, x_{3}\right) \quad\left(=-\omega\left(x_{1}, x_{2}, x_{3}\right)\right)
$$

holds.

We define $\Pi^{+}$and $\Pi^{-}$to be the set of all order-preseving and all orderreversing permutations of $\overline{\mathbb{F}}$ respectively. Finally we put $\mathrm{PGL}^{+}:=\mathrm{PGL}(2, \mathbb{F}) \cap \Pi^{+}$ and $\mathrm{PGL}^{-}:=\mathrm{PGL}(2, \mathbb{F}) \cap \Pi^{-}$. Then $\mathrm{PGL}(2, \mathbb{F})=\mathrm{PGL}^{+} \dot{\mathrm{P}} \mathrm{PGL}^{-}$.

Theorem 2.2 (Jakóbowski 1993). Let (F,P) be an half-ordered field and let $f, g \in \Pi^{+}$. Then $\Sigma(f, g):=\mathrm{PGL}^{+} \cup g^{-1} \mathrm{PGL}^{-} f$ acts sharply 3-transitively on $\overline{\mathbb{F}}$. 
Theorem 2.2 is a generalization of a result of C. Pedrini [10].

Let $(\mathbb{F}, \mathrm{P})$ be a half-ordered field and $f, g \in \Pi^{+}$. Then $\mathcal{H}(\mathbb{F}, f, g):=\mathcal{H}(\Sigma(f, g))$ is a hyperbola structure by Theorem 2.2.

For $a, b \in \mathbb{F}, a \neq 0$ let $a^{\bullet}: \overline{\mathbb{F}} \rightarrow \overline{\mathbb{F}}$ and $b^{+}: \overline{\mathbb{F}} \rightarrow \overline{\mathbb{F}}$ denote the canonic extension of the left multiplication $x \mapsto a x$ and translation $x \mapsto b+x$ respectively.

We note that $b^{+} \in \mathrm{PGL}^{+}$for all $b \in \mathbb{F}$ (cf. [13, 2.4]).

\section{Transitive groups of axial homologies of hyperbola structures}

Let $\mathcal{H}=\left(P, \mathfrak{K}, \mathfrak{G}_{1} \cup \mathfrak{G}_{2}\right)$ be a hyperbola structure. Let $\alpha \in$ Aut $\mathcal{H}$ and $G, H \in \mathfrak{G}$ with $G \cap H=\emptyset$. The automorphism $\alpha$ is called an axial homology with axis $G, H$ (shortly $(G, H)$-homology), if $G \cup H \subset$ Fix $\alpha$. The automorphism $\alpha$ is called a $G$-translation, if $\alpha=$ id or Fix $\alpha=G$.

If $\alpha$ is a $(G, H)$-homology or a $G$-translation with $G \in \mathfrak{G}_{i}$ then $\alpha(X)=X$ for all $X \in \mathfrak{G} \backslash \mathfrak{G}_{i}$. Note that every $(G, H)$-homology and every $G$-translation is a proper automorphism, i.e. maps $\mathfrak{G}_{i}$ onto $\mathfrak{G}_{i}$.

All $(G, H)$-homologies form a group $\mathrm{A}(G, H)$. The set of all $G$-translations is denoted by $\mathrm{T}(G)$.

Lemma 3.1. Let $\gamma \in \mathrm{A}(G, H)$ and $p \in P \backslash(G \cup H)$ with $\gamma(p)=p$. Then $\gamma=\mathrm{id}$.

Proof. We may assume $G, H \in \mathfrak{G}_{1}$. Consider $X:=[p]_{1}$. Then $X \neq G, H$. For $x \in X$ we have $\gamma(x)=\gamma\left([p]_{1} \cap[x]_{2}\right)=[\gamma(p)]_{1} \cap[x]_{2}=[p]_{1} \cap[x]_{2}=x$. Hence, for all $C \in \mathfrak{K}$ we have $\gamma(C)=C$, because $g:=C \cap G, h:=C \cap H, x:=C \cap X$ are three fixed points. Let $z \in P \backslash(G \cup H)$. Then there is a circle $C \in \mathfrak{K}$ with $z \in C$, and $\gamma(z)=\gamma\left(C \cap[z]_{2}\right)=\gamma(C) \cap[z]_{2}=C \cap[z]_{2}=z$.

Let $\Gamma$ be a subgroup of Aut $\mathcal{H}$. Then $\Gamma$ is called $(G, H)$-transitive, if there is an $X \in \mathfrak{G}$ with $|G \cap X|=1$ such that $\Gamma \cap \mathrm{A}(G, H)$ acts transitively on $X \backslash(G \cup H)$. Moreover, $\Gamma$ is called $G$-transitive, if there is an $X \in \mathfrak{G}$ with $|G \cap X|=1$ such that $\Gamma \cap \mathrm{T}(G)$ acts transitively on $X \backslash G$. The hyperbola structure is called $(G, H)$-transitive if Aut $\mathcal{H}$ is $(G, H)$-transitive.

If $\Gamma$ is $(G, H)$-transitive and $G \in \mathfrak{G}_{i}$ then $\Gamma \cap \mathrm{A}(G, H)$ acts transitively on $\mathfrak{G}_{i} \backslash\{G, H\}$, hence transitively on $Y \backslash(G \cup H)$ for every $Y \in \mathfrak{G} \backslash \mathfrak{G}_{i}$.

Looking for all imaginable configurations $\mathfrak{Z}:=\{G \in \mathfrak{G} \mid \Gamma$ is $G$-transitive $\}$ the subgroups $\Gamma$ of the automorphism group of a hyperbola structure were typified 
In the following we consider a hyperbola structure $\mathcal{H}=\mathcal{H}(M, \Sigma)$ with id $\in \Sigma$. To avoid brackets, for $x \in M$ we identify the point $(x, x)$ with $x$.

In the following lemma we characterize those permutations $\alpha, \beta$ of $M$ yielding an axial homology $\alpha \circ \grave{\beta}$.

Lemma 3.4. Let $g, h \in M, g \neq h$ and $G_{i}:=[g]_{i}, H_{i}:=[h]_{i}(i=1,2)$. For all bijections $\alpha, \beta: M \rightarrow M$ we have:

(1) $\alpha \circ \grave{\beta}$ is an axial homology $\Longleftrightarrow \beta \Sigma \alpha^{-1}=\Sigma, \mid$ Fix $\alpha|\geq 2$,$| Fix \beta \mid \geq 2$ and $\beta=\mathrm{id}$ or $\alpha=\mathrm{id}$.

(2) $\alpha$ is a $\left(G_{1}, H_{1}\right)$-homology $\Longleftrightarrow g, h \in$ Fix $\alpha$ and $\Sigma \alpha=\Sigma$. In particular we have: $\alpha \in \mathrm{A}\left(G_{1}, H_{1}\right) \Longrightarrow \alpha \in \Sigma_{g, h}{ }^{2}$.

(3) $\grave{\beta}$ is a $\left(G_{2}, H_{2}\right)$-homology $\Longleftrightarrow g, h \in \operatorname{Fix} \beta$ and $\Sigma=\beta \Sigma$. In particular we have: $\grave{\beta} \in \mathrm{A}\left(G_{2}, H_{2}\right) \Longrightarrow \beta \in \Sigma_{g, h}$.

Proof. Because of Lemma 2.1 we may assume $\beta \Sigma \alpha^{-1}=\Sigma$, i.e. $\beta \Sigma=\Sigma \alpha$. We have $G_{1}=\{g\} \times M, H_{1}=\{h\} \times M$. Hence

$$
\begin{aligned}
\dot{\alpha} \circ \grave{\beta} \in \mathrm{A}\left(G_{1}, H_{1}\right) & \Longleftrightarrow\{g\} \times M,\{h\} \times M \subset \operatorname{Fix} \dot{\alpha} \circ \grave{\beta} \\
& \Longleftrightarrow g, h \in \text { Fix } \alpha \text { and } \beta=\text { id. }
\end{aligned}
$$

Hence $\alpha \in \mathrm{A}\left(G_{1}, H_{1}\right)$ implies $\Sigma \alpha^{-1}=\Sigma$, thus $\Sigma \alpha=\Sigma$, and $\alpha=$ id $\circ \alpha \in \Sigma_{g, h}$. This proves (2) and the first part of (1). The remaining parts follow in the same way.

The meaning of the $(G, H)$-transitivity for the permutation set $(M, \Sigma)$ is given in the following lemma.

Lemma 3.5. Let $g, h \in M, g \neq h$ and $G_{i}:=[g]_{i}, H_{i}:=[h]_{i}(i=1,2)$ and

$$
\begin{aligned}
& A:=\left\{\alpha \in \operatorname{Sym} M \mid \dot{\alpha} \text { is a }\left(G_{1}, H_{1}\right) \text {-homology }\right\} \\
& B:=\left\{\alpha \in \operatorname{Sym} M \mid \grave{\alpha} \text { is } a\left(G_{2}, H_{2}\right) \text {-homology }\right\}
\end{aligned}
$$

Then we have:

(1) $A=A^{-1} \subset \Sigma_{g, h}$ and $\Sigma A=\Sigma$;

(2) Aut $\mathcal{H}$ is $\left(G_{1}, H_{1}\right)$-transitive $\Longleftrightarrow A=\Sigma_{g, h}$;

(3) $B=B^{-1} \subset \Sigma_{g, h}$ and $B \Sigma=\Sigma$;

(4) Aut $\mathcal{H}$ is $\left(G_{2}, H_{2}\right)$-transitive $\Longleftrightarrow B=\Sigma_{g, h}$.

\footnotetext{
${ }^{2} \Sigma_{g, h}$ denotes the stabilizer of $g$ and $h$ in $\Sigma$.
} 




\section{Hyperbola structures $\mathcal{H}(\mathbb{F}, f, g)$ with transitive groups of axial homologies}

Let $\mathcal{H}(\mathbb{F}, f, g)$ be a hyperbola structure over a half-ordered field $(\mathbb{F}, \mathrm{P})$. We consider the following sets of bijections:

$$
\begin{gathered}
M:=\{f \in \operatorname{Sym} \mathbb{F} \mid f(0)=0, \quad f(\infty)=\infty, \\
\qquad a \in P, \forall x \in \mathbb{F}: f(a x)=f(a) f(x)\}, \\
I:=\{f \in \operatorname{Sym} \mathbb{F} \mid f(0)=0, \quad f(\infty)=\infty, \\
\quad \forall d \in \mathbb{F} \backslash P, \forall x \in \mathbb{F}: f(d f(x))=f(d) x\}, \\
\Lambda^{+}:=M \cap I \cap \Pi^{+} .
\end{gathered}
$$

A permutation $f \in \operatorname{Sym} \mathbb{F}$ with $f(0)=0, f(\infty)=\infty$ is called a Moulton mapping if $\left.f\right|_{P}=\left.\mathrm{id}\right|_{P}$ and if there is a $k \in P$ such that for all $x \in \mathbb{F} \backslash P$, the equality $f(x)=k x$ holds.

Lemma 4.1. For every Moulton mapping $f$ we have:

(1) $f \in M \cap I$;

(2) $f \in \Pi^{+} \Longleftrightarrow \forall x \in \mathbb{F} \backslash P: \chi(1-x)=\chi(1-f(x))$.

Proof. (1) For $a \in P, d, x \in \mathbb{F} \backslash P$ we have $f(d f(a))=f(d a)=k d a=f(d) a$ and $f(d f(x))=f(k d x)=k d x=f(d) x$.

(2) “ $\Rightarrow$ ". For $x \in \mathbb{F} \backslash P$ we have $\chi(1-x)=-\omega(1, x, \infty)=-\omega(1, f(x), \infty)=$ $\chi(1-f(x))$.

"६". For $x, y \in \mathbb{F} \backslash P$ we have $\chi(x-y)=\chi(f(x)-f(y))$ because of $\chi(k)=1$. For $x \in P, y \in \mathbb{F}$ we have $\chi(x-y)=\chi(x) \chi\left(1-x^{-1} y\right)=$ $\chi(f(x)) \chi\left(1-f\left(x^{-1} y\right)\right)=\chi\left(f(x)-f(x) f\left(x^{-1} y\right)\right)=\chi(f(x)-f(y))$ by (1). Hence, for all $x, y \in \mathbb{F}, x \neq y$ holds $\chi(x-y)=\chi(f(x)-f(y))$. Thus $f \in \Pi^{+}$.

Examples 4.2. (1) Let $(\mathbb{F}, P)$ be an ordered field, i.e. $(\mathbb{F}, P)$ is a half-ordered field with $P+P \subset P$. Then every Moulton mapping $f$ is order-preserving by Lemma 4.1(2), hence $f \in \Lambda^{+}$.

(2) Let $\mathbb{F}=\mathbb{Q}$ be the field of rational numbers. There is exactly one subgroup $P<\mathbb{Q}^{*}$ of index 2 containing all primes different from 3 with $-1 \in P$ (cf. [11]). For $x \in \mathbb{Q}^{*}$ there exists $z \in \mathbb{Z}, n \in \mathbb{N}$ and $\nu \in \mathbb{Z}$ such that $x=3^{\nu} \frac{z}{n}$ and $3 \nmid z, n$, i.e. $z, n \in P$. Therefore $x=3^{\nu} \frac{z}{n} \notin P$ iff $\nu \in 2 \mathbb{Z}+1$. Take a $k \in P \cap \mathbb{N}$ with $3 \nmid k$ and consider the Moulton mapping $f$ defined 
by $k$. For $x=3^{\nu} \frac{z}{n}$ with $\nu \neq 0$ and $3 \nmid z, n$ we have

$$
\frac{1-x}{1-k x}=\frac{n-3^{\nu} z}{n-3^{\nu} z k}=\frac{3^{-\nu} n-z}{3^{-\nu} n-z k} \in P .
$$

Therefore $f$ is order-preserving by Lemma 4.1(2), thus $f \in \Lambda^{+}$.

Lemma 4.3. Let $(\mathbb{F}, P)$ be a half-ordered field and $f \in \Lambda^{+}$. Then we have:

(1) For all $x \in P: f^{2}(x)=x$;

(2) if there is an $x \in P$ with $f(x) \neq x$ then $-1 \in P$;

(3) if $\left.f\right|_{P}=\left.i d\right|_{P}$ then $f$ is a Moulton mapping;

(4) if $-1 \notin P$ then $f$ is a Moulton mapping;

(5) for $\Sigma=\mathrm{PGL}^{+} \cup \mathrm{PGL}^{-} f$ the stabilizer $\Sigma_{\infty, 0}$ contains at most one involution.

Proof. Since $f$ is order-preserving $f(P)=P$.

(1) Let $d \in \mathbb{F}^{*} \backslash P$. For all $x \in P$ we have $f(x) f(d)=f(x d)=f\left(d \cdot f\left(f^{-1}(x)\right)\right)=$ $f(d) \cdot f^{-1}(x)$, hence $f(x)=f^{-1}(x)$, i.e. $f^{2}(x)=x$.

(2) Let $x \in P, f(x) \neq x$. Then $\omega(x, f(x), \infty)=\omega(f(x), x, \infty)$ by (1), thus $\chi(x-f(x))=-\omega(x, f(x), \infty)=-\omega(f(x), x, \infty)=\chi(f(x)-x)$, hence $\chi(-1)=1$, i.e. $-1 \in P$.

(3) Consider a fixed $d \in \mathbb{F}^{*} \backslash P$. For every $x \in \mathbb{F}^{*} \backslash P$ there exists a $y \in P$ with $x=d y$, in particular there is a $k \in P$ with $f(d)=k d$. Hence $f(x)=$ $f(d y)=f(d f(y))=f(d) y=k d y=k x$.

(4) This follows by (2) and (3).

(5) For $\gamma \in \operatorname{PGL}(2, \mathbb{F})$ we have $\infty, 0 \in$ Fix $\gamma f$ if and only if $\infty, 0 \in$ Fix $\gamma$, hence $\Sigma_{\infty, 0}=\left\{a^{\bullet} \mid a \in P\right\} \cup\left\{d^{\bullet} f \mid d \in \mathbb{F}^{*} \backslash P\right\}$. First we characterize the involutions of $\Sigma_{\infty, 0}$.

For $a \in P$ we have: $\quad a^{\bullet}$ is an involution $\Longleftrightarrow a=-1 \neq 1$.

Indeed, $\left(a^{\bullet}\right)^{2}=$ id implies $1=a^{\bullet}\left(a^{\bullet}(1)\right)=a^{2}$, hence $a \in\{-1,1\}$, and conversely $1^{\bullet}=\mathrm{id}$ and $-1 \neq 1$ implies that $(-1)^{\bullet}$ is involutory.

For $d \in \mathbb{F}^{*} \backslash P$ we have: $\quad d^{\bullet} f$ is an involution $\Longleftrightarrow d f(d)=1$.

Indeed, $d^{\bullet} f \neq$ id and $\left(d^{\bullet} f\right)^{2}=$ id implies $1=d f(d f(1))=d f(d)$, and conversely $d f(d)=1$ implies for all $x \in \overline{\mathbb{F}}: \quad\left(d^{\bullet} f\right)^{2}(x)=d f(d f(x))=$ $d f(d) x=x$.

We have to distinguish two cases: $-1 \notin P$ and $-1 \in P$. 

Lemma 4.5. Let $P$ be a subgroup of $\left(\mathbb{F}^{*}, \cdot\right)$ of index 2 and let $f: \mathbb{F} \rightarrow \mathbb{F}$ be an additive bijection satisfying $f(a x)=f(a) f(x)$ for all $a \in P, x \in \mathbb{F}$. Then $f$ is an automorphism of $(\mathbb{F},+, \cdot)$.

Proof. Since $f$ is additive we have $f(-x)=-f(x)$, in particular $f(-1)=-1$, hence $f((-1) x)=f(-1) f(x)$. If $|\mathbb{F}|=3$ we have $f=$ id. Let $|\mathbb{F}|>4$. Consider $d \in \mathbb{F}^{*} \backslash P, d \neq 0,1$. Then $d+1 \in P$ or $d^{2}+d \in P$. Hence there is a $p \in P$ with $d+p \in P$. For all $x \in \mathbb{F}$ we have $f(d x)+f(p) f(x)=f(d x)+f(p x)=$ $f((d+p) x)=f(d+p) f(x)=f(d) f(x)+f(p) f(x)$, hence $f(d x)=f(d) f(x)$.

full screen

close

quit

Lemma 4.6. There exist $A, B \in \mathfrak{G}_{1}$, such that $\mathcal{H}(\mathbb{F}, f, g)$ is $(A, B)$-transitive if and only if there is an automorphism $\phi$ of $(\mathbb{F},+, \cdot)$ such that $f \in \phi \mathrm{PGL}^{+} \Lambda^{+} \mathrm{PGL}^{+}$, $g \in \phi \mathrm{PGL}^{+}$.

Proof. (a) Let $A=:\{a\} \times \overline{\mathbb{F}}$ and $B=:\{b\} \times \overline{\mathbb{F}}$ with $a \neq b$. Then there is an $\alpha \in \mathrm{PGL}^{+}$with $\alpha(a)=\infty, \alpha(b)=0$ and $\alpha$ is an isomorphism from $\mathcal{H}(\mathbb{F}, f, g)$ to $\mathcal{H}\left(\mathbb{F}, f \alpha^{-1}, g\right)$ (cf. $[13,2.1]$ ). By Lemma 3.2 the hyperbola structure $\mathcal{H}(\mathbb{F}, f, g)$ is $(A, B)$-transitive if and only if $\mathcal{H}\left(\mathbb{F}, f \alpha^{-1}, g\right)$ is $\left([\infty]_{1},[0]_{1}\right)$-transitive. By [13, Remark 2.5] there are $\rho, \sigma \in \mathrm{PGL}^{+}$such that $\{\infty, 0,1\} \subset$ Fix $\rho f \alpha^{-1} \cap$ Fix $\sigma g$, and we have $\mathcal{H}\left(\mathbb{F}, f \alpha^{-1}, g\right)=\mathcal{H}\left(\mathbb{F}, \rho f \alpha^{-1}, \sigma g\right)$.

(b) Let $\mathcal{H}(\mathbb{F}, f, g)$ be $(A, B)$-transitive. Put $\phi:=\sigma g \in \Pi^{+}$. By (a) the hyperbola structure $\mathcal{H}\left(\mathbb{F}, \rho f \alpha^{-1}, \phi\right)$ is $\left([\infty]_{1},[0]_{1}\right)$-transitive, and $\{\infty, 0,1\} \subset \operatorname{Fix} \phi$.

Consider $x \in \mathbb{F}^{*} \backslash P$. Then there exists an order-reversing bijection $\xi \in$ $\phi^{-1} \mathrm{PGL}^{-} \rho f \alpha^{-1}=\phi^{-1} \mathrm{PGL}^{-} f \alpha^{-1}$ with $\xi(0)=0, \xi(\infty)=\infty, \xi(1)=x$. By Lemma 3.5(2), $\xi$ is a $\left([\infty]_{1},[0]_{1}\right)$-homology, hence $\phi^{-1} \mathrm{PGL}^{-} f \alpha^{-1} \xi^{-1}=$ $\mathrm{PGL}^{+}$because of $\mathrm{PGL}^{+} \xi^{-1} \subset \Pi^{-}$. Thus there is a $\nu \in \mathrm{PGL}^{-}$such that $\xi=\phi^{-1} \nu f \alpha^{-1}$, hence $\mathrm{PGL}^{+}=\phi^{-1} \mathrm{PGL}^{-} \nu^{-1} \phi=\phi^{-1} \mathrm{PGL}^{+} \phi$.

Let $a, b \in \mathbb{F}$. Because of $a^{+}, b^{+} \in \mathrm{PGL}^{+}$we have $\phi^{-1} a^{+} \phi, \phi^{-1} b^{+} \phi \in \mathrm{PGL}^{+}$, and for $a \neq 0$ the mapping $\phi^{-1} a^{+} \phi$ has exactly $\infty$ as fixed point, hence $\phi^{-1} a^{+} \phi=\phi^{-1}(a)^{+}$because of $\phi^{-1} a^{+} \phi(0)=\phi^{-1}(a)$. Therefore,

$$
\begin{aligned}
\phi^{-1}(a+b) & =\phi^{-1}\left(a^{+}(b)\right)=\phi^{-1}\left(a^{+} \phi \phi^{-1}(b)\right)=\phi^{-1}(a)^{+}\left(\phi^{-1}(b)\right) \\
& =\phi^{-1}(a)+\phi^{-1}(b) .
\end{aligned}
$$

Hence $\phi^{-1}$ is additive.

For $a \in P$ we have $a^{\bullet}, \phi^{-1} a^{\bullet} \phi \in \mathrm{PGL}^{+}$, hence $\phi^{-1} a^{\bullet} \phi=\phi^{-1}(a)^{\bullet}$. Therefore, for all $z \in \mathbb{F}$ we have

$$
\begin{aligned}
\phi^{-1}(a z)=\phi^{-1}\left(a^{\bullet}(z)\right)=\phi^{-1} a^{\bullet}\left(\phi^{-1}(z)\right)=\phi^{-1}(a)^{\bullet} & \left(\phi^{-1}(z)\right) \\
& =\left(\phi ^ { - 1 } ( a ) \cdot \left(\phi^{-1}(z) .\right.\right.
\end{aligned}
$$

Hence $\phi$ is an automorphism of the field $\mathbb{F}$ by Lemma 4.5 . 

$d_{b}=\left(1-m_{b}\right) f(b)$, hence

$$
f(b+x)=f b^{+}(x)=d_{b}^{+} m_{b} f(b)^{+}(x)=m_{b}(f(x)+f(b))+\left(1-m_{b}\right) f(b)
$$

for all $x \in \mathbb{F}$, in particular $0=f(0)=m_{b}(f(-b)+f(b))+\left(1-m_{b}\right) f(b)=$ $m_{b} f(-b)+f(b)$, thus $m_{b}=-\frac{f(b)}{f(-b)}$. Because of $f \in \Lambda^{+}$we have

$$
m_{b}=-\frac{f(b)}{f(b) f(-1)}=-\frac{1}{f(-1)}
$$

full screen

close

for all $b \in P$. Hence for all $b \in P, x \in \mathbb{F}$ we have

$$
f(b+x)=-\frac{1}{f(-1)}(f(x)+f(b))+\left(1+\frac{1}{f(-1)}\right) f(b)=-\frac{1}{f(-1)} f(x)+f(b) .
$$

If $x \in P$ we obtain $f(b+x)=-\frac{1}{f(-1)} f(b)+f(x)$ by exchanging $b$ and $x$ in the previous formula. Therefore we have $\left(1+\frac{1}{f(-1)}\right) f(b)=\left(1+\frac{1}{f(-1)}\right) f(x)$ for all $b, x \in P$. Because of $|P| \geq 2$ this implies $1+\frac{1}{f(-1)}=0$, hence $f(-1)=-1$ and $m_{b}=1$ for all $b \in P$. For $b \in \mathbb{F}^{*} \backslash P$ we obtain

$$
m_{b}=-\frac{f(b)}{f(-b)}=-\frac{f(b)}{f(b f(-1))}=-\frac{f(b)}{f(b) \cdot(-1)}=1 .
$$

Therefore we have for all $b, x \in \mathbb{F}$ :

$$
f(b+x)=m_{b}(f(x)+f(b))+\left(1-m_{b}\right) f(b)=f(x)+f(b) .
$$

Hence $\left.f\right|_{\mathbb{F}}$ is an automorphism of $(\mathbb{F},+, \cdot)$ by Lemma 4.5 .

Consider $d \in \mathbb{F}^{*} \backslash P$. Since $f \in \operatorname{Aut}(\mathbb{F},+, \cdot) \cap I^{+}$we have for all $x \in \mathbb{F}$ : $f(d) x=f(d f(x))=f(d) f^{2}(x)$, hence $f^{2}(x)=x$.

$\left(\mathrm{ii}_{2}\right) \Rightarrow$ (iii). This follows from the fact that $\mathcal{H}(\mathbb{F}, f, g)$ and $\mathcal{H}(\mathbb{F}, g, f)$ are isomorphic (cf. $[13,2.2]$ ) by the implication (ii 1 ) $\Rightarrow$ (iii).

(iii) $\Rightarrow$ (i). Since $\left.f\right|_{\mathbb{F}}$ is an automorphism of $(\mathbb{F},+, \cdot)$ we have $\mathrm{PGL}^{-} f=f \mathrm{PGL}^{-}$, and because of $f^{2}=\mathrm{id}$ we obtain $\mathrm{PGL}^{-} f \cdot \mathrm{PGL}^{-} f=\mathrm{PGL}^{+}$. Therefore $\Sigma$ is a group.

Corollary 4.10. If $\Sigma=\mathrm{PGL}^{+} \cup \mathrm{PGL}^{-}$f is a group, then $\mathcal{H}(\mathbb{F}, f$, id $)$ is a Minkowski plane (cf. Hartmann [4]).

Corollary 4.11. If $\mathcal{H}(\mathbb{F}, f, g)$ admits an automorphism group of type $(1 ; 1)$ then $\mathcal{H}(\mathbb{F}, f, g)$ is of type $(4 ; 4)$.

Proof. By assumption there are subgroups $\Gamma_{1}, \Gamma_{2}$ of Aut $\mathcal{H}(\mathbb{F}, f, g)$ such that $\mathfrak{A}_{i}\left(\Gamma_{i}\right) \neq \emptyset$ for $i=1,2$. Since $\mathfrak{A}_{1}\left(\Gamma_{1}\right) \neq \emptyset$ we may assume that $g=\mathrm{id}$ and 

[2] Y. Chen and G. Kaerlein, Eine Bemerkung über endliche Laguerre- und Minkowski-Ebenen, Geom. Dedicata 2 (1973), 193-194.

[3] E. Hartmann, Beispiele nicht einbettbarer reeller Minkowski-Ebenen, Geom. Dedicata 10 (1981), 155-159.

[4]_, Minkowski-Ebenen mit Transitivitätseigenschaften, Results Math. 5 (1982), 136-148.

[5] W. Heise and H. Karzel, Symmetrische Minkowski-Ebenen, J. Geom. 3 (1973), 5-20.

[6] J. Jakóbowski, A full generalization of Moulton's method applied to Minkowski axioms, Zesz. Nauk. Geom. 20 (1993), 13-21.

[7] H. Karzel and H.-J. Kroll, Perspectivities in circle geometries, in Geometry - von Staudt's point of view, Proc. NATO Adv. Study Inst., Bad Windsheim/Ger. 1980 (K. Strambach \& P. Plaumann, eds.), 1981, pp. 51-99.

[8] M. Klein, Classification of Minkowski planes by transitive groups of homotheties, J. Geom. 43 (1992), 116-128.

[9] M. Klein and H.-J. Kroll, A classification of Minkowski planes, J. Geom. 36 (1989), 99-109.

[10] C. Pedrini, 3-reti (non immergibili) aventi dei piani duali di quelli di Moulton quali sottopiani, Atti Accad. Naz. Lincei Rend. Cl. Sci. Fis. Mat. Natur. 40 (1966), 385-392.

[11] E. Sperner, Beziehungen zwischen geometrischer und algebraischer Anordnung, Sitzungsber. Heidelb. Akad. Wiss. Math.-Natur. Kl. 10 (1949), 413-448.

[12] G. F. Steinke, A family of Minkowski planes over half-ordered fields, Beitr. Algebra Geom. 37 (1996), 355-366.

[13] _ A classification of Minkowski planes over half-ordered fields, J. Geom. 69 (2000), 192-214.

[14] H. Wefelscheid, Über die Automorphismengruppen von Hyperbelstrukturen, in Beitr. geom. Algebra, Proc. Symp. Duisburg 1976, 1977, pp. 337-343. 


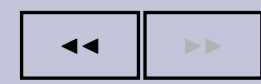

4

page $18 / 18$

go back

full screen

close

quit
Hans-Joachim Kroll

Zentrum Mathematik, TU MÜnchen, D-80290 MÜnChen, Germany

e-mail: kroll@ma.tum.de

Andrzej Matraś

Department of Mathematics and Informatics, UWM OlSZTYN, ŻoŁnierska 14, PL-10-561 OLSZTYN, POLAND

e-mail: matras@uwm.edu.pl 